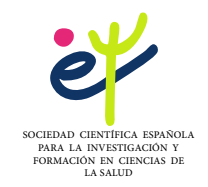

\title{
El uso del teléfono móvil en clase y su efecto sobre el engagement académico y el agotamiento: Un estudio de diario en estudiantes universitarios
}

\section{The use of the mobile phone in class and its effect on academic engagement and burnout: A journal study in university students}

\author{
Alfredo Rodríguez Muñoz* (D) y Mirko Antino \\ Universidad Complutense de Madrid, Madrid, España
}

\section{RESUMEN}

En los últimos ańos, el uso de nuevas tecnologías ha crecido de manera exponencial. Actualmente, está presente en todos los ámbitos de nuestra vida, incluido el académico. El presente estudio examinó el efecto del uso diario del teléfono inteligente durante las clases tanto en el engagement académico como en el agotamiento. Se recolectaron datos de 45 estudiantes universitarios con un diseńo de diario (cinco días hábiles consecutivos) dos veces al día ( $\mathrm{N}=450$ ocasiones). Los análisis multinivel mostraron que el uso diario del teléfono inteligente durante las clases predijo positivamente el nivel de agotamiento. Además, encontramos que el uso del teléfono inteligentes en clase tiene efectos negativos en el engagement de los estudiantes. Los resultados se pueden utilizar para mejorar el engagement y reducir el agotamiento en estudiantes universitarios.

Palabras clave: smartphone, engagement, exhaustion, university students.

Para correspondencia: Correo: Alfredo Rodríguez Muñoz Universidad Complutense de Madrid, Madrid, Espańa. Email: alfredo.rodriguez@psi.ucm.es 


\begin{abstract}
In recent years, the use of new technologies has grown exponentially. Currently, it is present in all areas of our life, including the academic environment. The present study investigated the effect of daily use of smartphone during classes on both academic engagement and exhaustion. Data were collected from 45 university students with a daily diary design (five consecutive working days) twice a day $(\mathrm{N}=450$ occasions). Multilevel analyses showed that the daily use of smartphone during classes positively predicted exhaustion afternoon. In addition, we found that the use of smartphone at class have negative effects on student's engagement. The results can be used to enhance engagement and reduce exhaustion in colleges and universities.
\end{abstract}

Keywords: smartphone, engagement, exhaustion, university students.

\title{
INTRODUCCIÓN
}

El "smartphone" o teléfono inteligente se ha convertido en un elemento indispensable en nuestras vidas. El uso de las nuevas tecnologías móviles ha crecido de forma exponencial en los últimos ańos. Las posibilidades que nos ofrecen las nuevas tecnologías móviles han modificado la forma que tenemos de trabajar, relacionarnos y entretenernos. En el ámbito académico la irrupción de la tecnología ha mejorado numerosos procesos. No obstante, también han aparecido diversos riesgos y problemáticas asociadas al uso de la misma. Por ejemplo, el uso excesivo de teléfonos inteligentes tiene, posiblemente, el potencial para convertirse en un comportamiento adictivo que puede interferir en nuestra salud y bienestar (Duke y Montag, 2017). Si bien la comunidad científica debate en la actualidad si esta dependencia debe o no considerarse un trastorno clínico, lo que resulta evidente es que presenta una sintomatología similar a la de otras conductas adictivas: uso excesivo, síndrome de abstinencia (sentimientos de ansiedad o irritabilidad ante la imposibilidad de utilizar las nuevas tecnologías), tolerancia (necesidad creciente de aumentar el tiempo de utilización de la tecnología para sentirse satisfecho) y consecuencias negativas en la vida de la persona (aislamiento social, incumplimiento de las responsabilidades personales, etcétera).

En este sentido, los teléfonos inteligentes pueden distraernos hasta tal punto en el que no podamos lograr un estado de concentración óptimo (Montag y Walla, 2016). Alton et al. (2014) encontraron que interrupciones tan breves como $2.8 \mathrm{~s}$ interrumpían el flujo de concentración de los participantes en su estudio y se asociaban con un mayor número de errores en tareas cognitivas. De esta forma, los teléfonos inteligentes con sus señales visuales y acústicas que alertan al propietario sobre información entrante actúan como 
interruptores, que tienen el potencial de interferir con la concentración y la productividad. Además, el refuerzo intermitente recibido por parte de los teléfonos inteligentes puede facilitar el desarrollo de un "hábito de verificación", es decir, inspecciones breves y repetidas del teléfono para buscar contenido nuevo (Duke y Montag, 2017).

Esta hiperconectividad puede asociarse también a problemas para la salud, debido a que conlleva una hiperactivación fisiológica y psicológica. Por ejemplo, Lanaj, Johnson, y Barnes (2014), en un estudio mediante el método de muestreo de experiencias, hallaron que el uso del smartphone por la noche reducía la calidad de sueño, lo que aumentaba el agotamiento a la mañana siguiente. En la misma línea, se han encontrado relaciones negativas del uso del teléfono de empresa con el distanciamiento psicológico (Derks et al., 2014) y la relajación diaria (Derks, ten Brummelhuis, Zecic, y Bakker, 2014).

Esta disminución de la energía disponible, puede relacionarse directamente con dos constructos que muestran mucha relación con lo energético; burnout y engagement. En general, el engagement se define como un estado mental positivo y activo que se distingue por el vigor, la dedicación y la absorción en el desempeño de tareas (Schaufeli et al., 2002). En este estado de engagement los estudiantes están psicológicamente presentes cuando desempeñan sus roles y son capaces de aprovechar todo su potencial. Además, muestran una actitud más positiva hacia su organización y una conexión emocional hacia la misma, siendo más productivos (Reio \& Sanders-Reio, 2011). En cuanto al "burnout", vulgarmente conocido como "estar quemado", es un constructo que alude principalmente a situaciones de agotamiento emocional.

Para explicar los efectos perjudiciales del uso del smartphone se suele utilizar el modelo de "esfuerzo-recuperación" de Meijman y Mulder (1998). Según este modelo, la realización de un esfuerzo (p.ej. multitarea en clase), tiene una serie de costes a corto plazo, tanto a nivel psicológico como fisiológico. De este modo, existen reacciones normales que se relacionan inevitablemente con el esfuerzo realizado (como la fatiga). Normalmente, estas reacciones no tienen por qué suponer un problema, y una vez que se realiza un descanso, los sistemas psicobiológicos se reestabilizan. Sin embargo, estas reacciones pueden conducir a problemas crónicos si existe una activación prolongada. Por lo tanto, a través de la recuperación, los sistemas psicofisiológicos activados vuelven a la línea base, evitándose así un proceso acumulativo que termina en problemas de fatiga crónica, estrés crónico, o problemas persistentes de sueño. Basándonos en estos argumentos, se hipotetiza que:

Hipótesis 1: El uso diario del teléfono móvil se relacionará negativamente con el nivel de engagement.

Hipótesis 2: El uso diario del teléfono móvil se relacionará positivamente con el nivel de agotamiento al final del día. 


\section{MÉTODO}

\section{Muestra y procedimiento}

La muestra del estudio estuvo formada por estudiantes de la Universidad Complutense de Madrid. Los participantes fueron reclutados a través de las redes sociales del investigador principal y de distintos colaboradores. Se empleó un diseño de diario con dos niveles de análisis (Nivel 1: Día; Nivel 2: Persona), con recogida de datos durante cinco días laborables consecutivos, dos veces al día. En concreto, el estudiante debía rellenar el cuestionario al salir de clase (tarde) y al finalizar el día (noche). Anteriormente, los participantes tenían que haber rellenado un cuestionario general, donde se incluía información socio-demográfica y los niveles generales de las variables. El enagement y el agotamiento se evaluaron al final de cada día, mientras que el uso del teléfono móvil en clase se registró al terminar la jornada de clases. Los protocolos de los distintos cuestionarios se unieron mediante un código personal. Los cuestionarios se enviaban directamente al investigador principal.

Se enviaron 80 protocolos, y se recibieron 45 (tasa de respuesta 56\%). La muestra final del estudio consistió en 35 mujeres $(77,7 \%)$ y 10 hombres $(23,3 \%)$. La edad media fue de 20,1 años (DT=2,03). La mayoría de los participantes eran estudiantes de psicología y trabajo social.

\section{Instrumentos}

Uso del teléfono móvil en clase. Los niveles diarios de uso del teléfono móvil en clase se evaluaron mediante la versión adaptada para estudios de diario de la escala de Uso Intensivo del teléfono móvil de Derks y Bakker (2014). La escala incluye tres ítems (p. ej., "Hoy en clase, no he podido resistirme a revisar mi smartphone del trabajo cuando me indicaba la llegada de nuevos mensajes"). La escala de respuesta consta de seis alternativas desde 1 ("No es cierto") a 6 ("Completamente cierto"). A mayor puntuación, mayor uso diario del teléfono por motivos laborales tras la jornada laboral. La media de los cinco días del coeficiente de fiabilidad de Cronbach fue de .71.

Engagement académico. Los niveles diarios de engagement se evaluaron mediante la versión adaptada para estudios de diario (Breevaart, Bakker, Demerouti, y Hetland, 2012) de la escala Utrecht de Engagement (Schaufeli, Salanova, González-Romá y Bakker, 2002). En el presente estudio nos centramos en lo que se consideran elementos clave del constructo, a saber, el vigor y la dedicación (González-Romá, Schaufeli, Bakker, y Lloret, 2006). La escala incluye tres ítems para vigor (p. ej., "Hoy, Mis tareas como estudiante me han hecho sentir lleno de energía”), y tres para dedicación (p. ej., "Hoy mis estudios me han inspirado"). La escala de respuesta consta de seis alternativas desde 1 ("No es cierto") a 6 ("Completamente cierto"). A mayor puntuación, mayor nivel de engagement. La media de los cuatro días del coeficiente de fiabilidad de Cronbach fue de .84 . 
Agotamiento. El agotamiento diario se evaluó mediante tres ítems de la prueba Maslach Burnout Inventory-General Survey (MBI-GS) propuesta por Schaufeli et al. (1996). Un ejemplo de ítems es "Hoy me he sentido emocionalmente agotado por mis estudios". Se utilizó una escala de respuesta de seis alternativas desde 1 ("No es cierto") a 6 ("Completamente cierto”). A mayor puntuación, mayor nivel de agotamiento. La media de los cuatro días del coeficiente de fiabilidad de Cronbach fue de 0.82

\section{Análisis de datos}

Nuestro conjunto de datos se compone de dos niveles de análisis. En concreto, las medidas repetidas en el mismo día constituyen el primer nivel (intra-individual), y las diferencias intersujeto el segundo nivel. Para examinar las hipótesis se llevaron a cabo análisis multinivel con estructuras jerárquicas mediante el programa estadístico MLwiN 3.02 (Rasbash, Steele Browne y Goldstein, 2017), con dos niveles: día (Nivel 1, N = 450), persona (Nivel 2, $\mathrm{N}=45$ participantes). Este modelo considera la persona como la unidad mayor de análisis, con los días agrupados por personas. Las variables del nivel 2 se centraron en torno a las puntuaciones medias generales (grand mean) y las del nivel 1 respecto a las puntuaciones medias de cada sujeto individualmente.

\section{RESULTADOS}

En la tabla 1 se muestran las medias, desviaciones típicas y correlaciones de las variables del estudio. Los análisis de correlación indican que el uso del teléfono móvil en clase trabajo se relaciona positivamente el agotamiento diario $(r=.24, \mathrm{p}<.01)$, y negativamente con el engagement $(\mathrm{r}=-.17, \mathrm{p}<.01)$. También se observó que el género y la edad se relacionaban con estas variables, por lo que se incluyeron como controles en todos los análisis posteriores. Para examinar si existía suficiente variabilidad intra-inter sujeto para la utilización de un modelo de dos niveles (personas, días), se calcularon las correlaciones intraclase con el modelo nulo para las variables del estudio. Los resultados mostraron que un modelo de dos niveles explica una cantidad significativa de varianza en cada uno de los niveles. En concreto, los resultados mostraron que el $59,8 \%$ de la varianza del engagement puede explicarse por las variaciones entre días, y el $40,2 \%$ de la varianza es atribuible a las variaciones entre personas. Respecto al agotamiento se observa que el $42,3 \%$ de su varianza se explica por las variaciones entre días, mientras que el $57,7 \%$ por las variaciones entre personas. Estos resultados apoyan la utilización de modelos multinivel con dos niveles de análisis, debido a que la varianza atribuido al nivel diario fue en todos los casos significativa. 


\section{Tabla 1}

Estadisticos descriptivos y correlaciones ( $N=45$ Participantes, $N=450$ Observaciones)

\begin{tabular}{lcccc}
\hline Variable & $\mathrm{M}(\mathrm{SD})$ & 1 & 2 & 3 \\
\hline Uso diario del teléfono móvil & $4.15(0.98)$ & --- & --- &.-- \\
2. Engagement diario & $3.81(1.05)$ & $-.17^{* *}$ & $-.45^{* *}$ & \\
3. Agotamiento diario & $2.75(1.11)$ & $.24^{* *}$ & & \\
\hline
\end{tabular}

${ }^{*} p<.05 .{ }^{* *} p<.01$.

\section{Tabla 2}

Modelo Multinivel. ( $N=45$ Participantes, $N=450$ Observaciones)

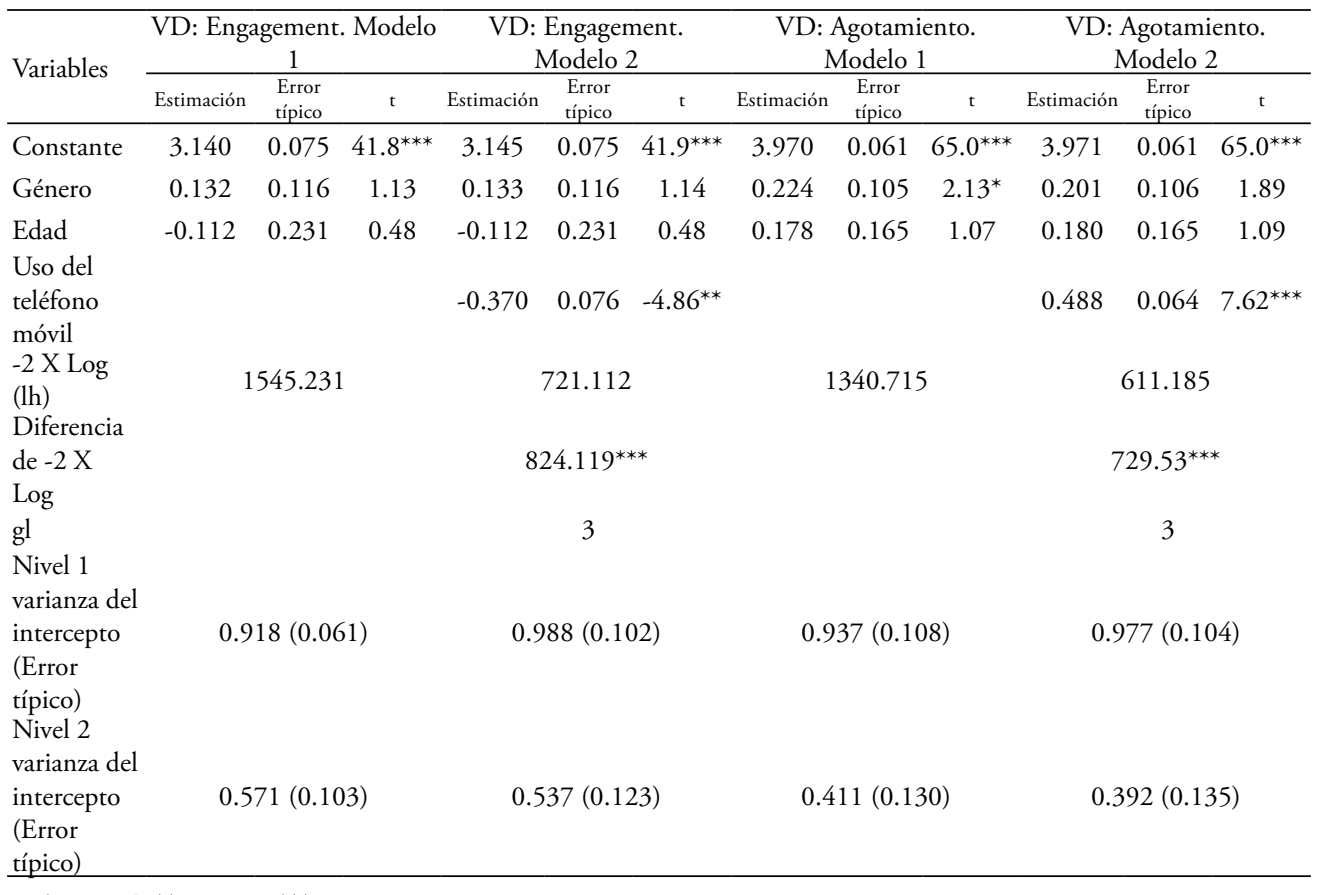

${ }^{*} p<.05 .{ }^{* *} p<.01 .{ }^{* * *} p<.001$.

Nota: Nivel 1 = Día; Nivel 2 = Persona.

La hipótesis 1 plantea que el nivel de uso diario del teléfono móvil predecirá negativamente el nivel de engagement académico diario. Para poner a prueba dicha hipótesis se examinaron dos modelos multinivel. En el Modelo 1, se incluía la constante como predictor, al igual que las variables control (género y edad). En el Modelo 2, se incluyó el nivel diario del uso de teléfono móvil. Los resultados apoyan la hipótesis 1, ya que el uso diario del teléfono móvil 
durante las clases se relaciona negativamente con los niveles diarios de conflicto trabajofamilia $(\gamma=-0.370, \mathrm{SE}=0.076, \mathrm{t}=-4.86, \mathrm{p}<.01)$.

La hipótesis 2 sugiere que existirá una relación positiva entre el uso del teléfono móvil y el nivel de agotamiento diario. Para examinar estas hipótesis se examinaron dos modelos multinivel. En el Modelo nulo, se incluía la constante y las variables control (información socio-demográfica) como predictores. En el Modelo 2, se incluyó el uso diario del teléfono móvil. Los resultados apoyan la hipótesis 2, ya que el uso diario del teléfono móvil se relaciona positivamente con los niveles diarios de agotamiento $(\gamma=0.488, \mathrm{SE}=0.064, \mathrm{t}=$ $7.62, \mathrm{p}<.01)($ Tabla 2$)$

\section{DISCUSIÓN}

La presente investigación muestra el efecto perjudicial del uso del teléfono móvil en horario lectivo. Una de las contribuciones de esta investigación es que seguimos la recomendación de Dormann y Griffin (2015) quienes plantean la necesidad de llevar a cabo investigaciones utilizando intervalos temporales cortos, o como ellos han denominado "shortitudinal studies", ya que aumenta la posibilidad de detectar efectos significativos. Desde un punto de vista metodológico, se examinan las variables desde una metodología de diario. Se ha demostrado que tanto el engagement como el agotamiento están sujetos a enormes fluctuaciones, incluso diarias (p. e.j., Breevart et al., 2012). Una ventaja importante de los estudios de diario es que minimizan el intervalo de tiempo que transcurre entre la experiencia de un evento y el auto-informe de esa situación, lo que reduce el sesgo retrospectivo y aumenta su validez.

En términos generales, nuestros datos confirman estudios previos que mostraban el efecto pernicioso del móvil en el burnout (Derks et al., 2016), la energía (Lanaj et al., 2014), y el distanciamiento psicológico (Derks et al., 2014). Del mismo modo, este estudio muestra que la multitarea es mala para el engagement académico y el agotamiento. Los hallazgos del presente estudio van en la línea del reciente estudio de Madore et al. (2020), donde se halló que realizar dos o más actividades digitales al mismo empeora la atención de los individuos y genera fallos en la memoria. En este sentido, los avances tecnológicos han facilitado a los individuos mantenerse conectados de manera constante. Sin embargo, la incapacidad para desconectar tiene consecuencias negativas en términos de recuperación y agotamiento (von Thiele Schwarz, 2011).

La explicación más directa de nuestros resultados se puede encontrar en el esfuerzo ańadido a la hora de realizar varias tareas simultáneamente. Según el modelo el Modelo de Esfuerzo-Recuperación (Meijman y Mulder, 1998), la realización de un esfuerzo tiene una serie de costes a corto plazo, tanto a nivel psicológico como fisiológico. Normalmente, estas 
reacciones no tienen por qué suponer un problema, y una vez que se realiza un descanso, los sistemas psicobiológicos se reestabilizan. Sin embargo, estas reacciones pueden conducir a problemas crónicos si existe una activación prolongada. En este sentido, si las reacciones de estrés o activación se prolongan durante un período más extenso de tiempo, manteniéndose la activación simpática, la recuperación es incompleta (Geurts y Sonnentag, 2006). Cuando existe esta falta de recuperación es posible entrar en un círculo vicioso, ya que la persona comienza el día en un estado psicofisiológico subóptimo, teniendo que realizar un esfuerzo adicional para contrarrestarlo y que su rendimiento no se vea afectado. De este modo, se produce un efecto acumulativo que termina en problemas de salud crónicos, acentuándose por el uso del móvil.

Antes de comentar las implicaciones prácticas del estudio, deben ser tenidas en cuenta algunas limitaciones de este estudio. En primer lugar, una posible limitación está relacionada con la estrategia de diseño. Aunque los estudios de diario ofrecen numerosas ventajas respecto a otros diseños, como la reducción del sesgo de respuesta o la posibilidad de tener en cuenta las fluctuaciones diarias de las variables objeto de estudio, no permiten conclusiones sobre efectos a largo plazo. Por lo tanto, la investigación futura también debería explorar el efecto a largo plazo de las consecuencias negativas del uso del teléfono inteligente. Otra posible limitación radica en que evaluamos el uso intensivo de teléfonos inteligentes, a través de una escala auto-informada. Sin embargo, para obtener una visión más precisa, sería necesario otro tipo de información, como datos objetivos sobre la frecuencia de uso o un libro de registro.

Las implicaciones prácticas del presente trabajo son claras. La primera de ellas es que a pesar de las ventajas en términos de flexibilidad y productividad que han mostrado las nuevas tecnologías, también acarrean costes para la salud. Estamos, por tanto, ante "un arma de doble filo". Nuestros hallazgos indican que los centros educativos deberían regular y/o reducir el uso del móvil dentro del aula, ya que supone un claro distractor y mina el compromiso y la energía del alumnado.

\section{Agradecimientos}

Esta investigación está financiada por el proyecto INNOVA-DOCENCIA UCM $\left(\mathrm{n}^{\circ} 209\right)$.

\section{REFERENCIAS}

Altmann, E. M., Trafton, J. G., \& Hambrick, D. Z. (2014). Momentary interruptions can derail the train of thought. Journal of Experimental Psychology: General, 143(1), 215226. https://doi.org/10.1037/a0030986 
Breevaart, K., Bakker, A. B., Demerouti, E., \& Hetland, J. (2012). The measurement of state work engagement: A multilevel factor analytic study. European Journal of Psychological Assessment, 28, 305-312. https://doi.org/10.1027/1015-5759/a000111

Derks, D., \& Bakker, A. B. (2014). Smartphone use, work-home interference, and burnout: A diary study on the role of recovery. Applied Psychology, 63, 411-440. https://doi. org/10.1111/j.1464-0597.2012.00530.x

Derks, D., Bakker, A. B., Peters, P., \& van Wingerden, P. (2016). Work-related smartphone use, work-family conflict and family role performance: The role of segmentation preference. Human Relations, 69, 1045-1068. https://doi. org/10.1177/0018726715601890

Derks, D., van Mierlo, H., \& Schmitz, E. B. (2014). A diary study on work-related smartphone use, psychological detachment and exhaustion: Examining the role of the perceived segmentation norm. Journal of occupational health psychology, 19, 7484. https://doi.org/10.1037/a0035076

Dormann, C., \& Griffin, M. A. (2015). Optimal time lags in panel studies. Psychological Methods, 20, 489-505. https://doi.org/10.1037/met0000041

Duke, É., \& Montag, C. (2017). Smartphone addiction, daily interruptions and self-reported productivity. Addictive behaviors reports, 6, 90-95. https://doi.org/10.1016/j. abrep.2017.07.002

González-Romá, V., Schaufeli, W. B., Bakker, A. B. \& Lloret, S. (2006). Burnout and work engagement: Independent factors or opposite poles? Journal of Vocational Behavior, 62, 165-174. https://doi.org/10.1016/j.jvb.2005.01.003

Lanaj, K., Johnson, R. E., \& Barnes, C. M. (2014). Beginning the workday yet already depleted? Consequences of late-night smartphone use and sleep. Organizational Behavior and Human Decision Processes, 124, 11-23. https://doi.org/10.1016/j. obhdp.2014.01.001

Madore, K. P., Khazenzon, A. M., Backes, C. W., Jiang, J., Uncapher, M. R., Norcia, A. M., \& Wagner, A. D. (2020). Memory failure predicted by attention lapsing and media multitasking. Nature, 1-5. https://doi.org/10.1038/s41586-020-2870-z

Meijman, T. F. y Mulder, G. (1998). Psychological aspects of workload. En P. J. Drenth, H. Thierry, \& C. J. de Wolff (Eds.), Handbook of Work and Organizational Psychology (2 ed., pp. 5-33). Hove, UK: Erlbaum.

Montag, C., \& Walla, P. (2016). Carpe diem instead of losing your social mind: Beyond digital addiction and why we all suffer from digital overuse. Cogent Psychology, 3(1), 1157281. https://doi.org/10.1080/23311908.2016.1157281 
Rasbash J, Steele F, Browne W, \& Goldstein H (2017). A User's Guide to MLwiN v3.00. Center for Multilevel Modelling: University of Bristol.

Reio Jr, T. G., \& Sanders-Reio, J. (2011). Thinking about workplace engagement: Does supervisor and coworker incivility really matter?. Advances in Developing Human Resources, 13(4), 462-478. https://doi.org/10.1177/1523422311430784

Schaufeli, W. B., Salanova, M., González-Romá, V., \& Bakker, A. B. (2002). The measurement of engagement and burnout and: a confirmative analytic approach. Journal of Happiness Studies, 3, 71-92. https://doi.org/10.1023/A:1015630930326

von Thiele Schwarz, U. (2011). Inability to withdraw from work as related to poor next-day recovery and fatigue among women. Applied Psychology: An International Review, 60, 377-396. https://doi.org/10.1111/j.1464-0597.2011.00440.x

Recibido: 23 de septiembre de 2020

Aceptado: 09 de noviembre de 2020 